\title{
Berth Allocation and Quay Crane Assignment Under Uncertainties
}

\author{
Caimao TAN, Junliang $\mathrm{HE}^{1}$ and Yuancai WANG \\ China Institute of FTZ Supply Chain, Shanghai Maritime University, Shanghai, \\ 201306, China
}

\begin{abstract}
The integration of berth allocation problem (BAP) and quay crane assignment problem (QCAP) is an cardinal seaside operations planning, which is susceptible to uncertainties, e.g. uncertain vessels arrival and maritime market. This paper addresses the integrated optimization of BAP and QCAP under uncertainties. A stochastic programming model is formulated for minimizing the waiting time and delay departure time of vessels. Besides, numerical experiments and scenario analysis are conducted to validate the effectiveness of the proposed model.
\end{abstract}

Keywords. container terminal, stochastic programming, B\&QCAP, genetic algorithm, uncertainty

\section{Introduction}

Maritime transportation plays an important role in driving economy growth and energizing the process of globalization, since its trade volume accounts for four fifths of the world's total merchandise trade. As the core node of maritime transportation, the efficiency of container terminal will directly affect the operation of maritime transportation. A total amount of the throughput of global container ports has achieved 802 million TEU in 2019, and it's expected to reach 973 million in 2023, according to the prediction of Drewry Shipping Consultants [1]. This means the throughput of global container ports is on the verge of a new billion era. Moreover, with increase of container vessel size, container ports are encountering another challenger, i.e. the repaid handling for mega container vessels [2].

BAP and QCAP are fundamental problems in optimizing container terminal operations, because berths and quay cranes (QCs) are the most critical resources in the front of the seaside. In particular, the integration of berth allocation and quay crane assignment secure an import position in efficient operation of container ports. Essentially, this integrated problem belongs to the intersection between the management and operations research. Up to present, plenty of studies were attempted in the integrated optimization of berth allocation and quay crane assignment in the static and deterministic environment. For a comprehensive overview, we refer to review the work given by $[3,4]$. However, most of the assumptions will hardly satisfied, e.g. the vessel arrival time and handling time. On the one hand, the delay of vessel arrival often occurs in the actual operation of the ports, and more than $40 \%$ of the international liners will be delayed at least one day [5]. On the other hand, the demand of maritime market has never been unchangeable, the global economic and trade, political environment and other factors have brought uncertainty to the maritime market. These uncertain events, not only made

\footnotetext{
1 Corresponding Author, Mail: caimaotan@shmtu.edu.cn.
} 
it difficult to determine the key parameters which are necessary for planning of berth allocation and quay crane assignment, but also lead to the baseline schedule cannot be implemented.

There are usually two strategies for coping with uncertainties: proactive and reactive strategy [6]. This paper covers both two strategies for the integrated optimization of BAP and QCAP under uncertainties. A stochastic programming model is formulated for minimizing the waiting time and delay of vessel departure. Besides, numerical experiments and scenario analysis are conducted to validate the effectiveness of the proposed model.

\section{Related works}

For the integration of BAP and QCAP (B\&QCAP), there are many related works from different aspects, e.g. deterministic or uncertain, discrete or continuous berth, timeinvariant or time variant QC assignment policy, maximizing handling efficiency or tradeoff between efficiency and energy consumption, et al. In this section, we mainly reviews the studies which are highly related to the strategies for coping with the integration of BAP and QCAP under uncertainties.

Han et al. [7] studied integration of BAP and QCAP with uncertainty of container handling time and dynamic vessel arrival with different service priorities, a mixed integer programming (MIP) model is established, and a simulation based genetic algorithm search procedure is applied to generate a perturbation insensitive robust schedule proactively. Considering stochastic arrivals, Hendriks et al. [8] developed a MIP to construct a robust window-based cyclic berth plan with minimally required crane capacity in the worst vessel arrival scenario. Goliasa et al. [9] presented a mathematical model and a solution approach for the discrete berth scheduling problem, where vessel arrival and handling times are not known with certainty (given the lower and upper bounds). A robust berth schedule by minimizing the average and the range of the total service times required for serving all vessels was provided. Rodriguez-Molins et al. [10] introduced the robustness of B\&QCAP by means of buffer times, which should be maximized to absorb possible incidences or break downs. To handle the uncertainty of QC productivity, the mean values of QCs were used by Shang et al. [11], and two robust models (robust optimization model and robust optimization model with price constraints) were proposed.

However, when disruptions occurred and the baseline schedule will no longer be the optimal solution, the reactive strategy should be implemented consequently. Zeng et al. [12] addressed the problem of recovering berth and quay crane schedule, the QC rescheduling strategy and berth reallocation strategy are proposed to tackle disruptions and recover the berth and QC schedule, and models for the two strategies are developed respectively. Li et al. [13] proposed a reactive recovery strategy which adjust the initial plan to handle realistic disruptions. The new berthing positions for vessels are restricted within a certain space. Quay cranes are allowed to move to other vessels before finishing current assigned vessels. Vessels requiring early dispatch are particularly considered in recovery planning. Four kinds of disruption, i.e., deviation of vessels' arrival times, deviation of vessels' operation times, calling of unscheduled vessels, and breakdown of QCs, were addressed by Xi et al. [14], and a reactive strategy was proposed, which takes the baseline schedule as the reference schedule, to deal with disruptions and minimize recovery cost. A rolling horizon heuristic is presented to derive good feasible solutions. 


\section{Model formulation}

\subsection{Notation definition}

Sets and parameters: $V$ represents the set of all vessels; $T$ represents the set of all periods; $Q$ represents the set of all QCs; $B$ represents the total number of berth segments; $\Omega$ represents the set of all future scenarios; $M$ represents a large positive number; $E T A_{i}$ represents the estimated arrival time of vessel $i$; ETD $D_{i}$ represents the estimated departure of vessel $i ; R_{i}^{\max }$ represents the maximum number of QCs that can be assigned to vessel $i$; $R_{i}^{\text {min }}$ represents the minimum number of QCs that should be assigned to vessel $i ; m_{i}$ represents the handling volume of vessel $i$ (units: TEU); $b_{i}$ represents the desired berthing position of vessel $i$; $\gamma_{q}$ represents the handling efficiency of a QC per unit time when $q$ QCs simultaneously serve the same vessel (units: move/h); $\xi$ represents the handling efficiency of a QC (units: $\mathrm{h} /$ move); $\mu$ represents the average value of containers where a QC handled per move (units: TEU/move); $L$ represents the length of the wharf; $l_{i}$ represents the length of vessel $i$, including horizontal safe distance; $p(w)$ represents the probability of scenario $w ; E T A_{i}(w)$ represents the estimated arrival time of vessel $i$ in scenario $w ; \operatorname{ETD}_{i}(w)$ represents the estimated finishing time of vessel $i$ in scenario $w ; R_{i}^{\max }(w)$ represents the maximum number of QCs that can be assigned to vessel $i$ in scenario $w ; R_{i}^{\min }(w)$ represents the minimum number of QCs that should be assigned to vessel $i$ in scenario $w ; m_{i}(w)$ represents the handling volume of vessel $i$ in scenario $w$ (units: TEU).

Decision variables: $s_{i}$ represents the start berthing time of vessel $i$; $\mathrm{y}_{\mathrm{i}}$ represents the actual berthing position of vessel $i$; $\mathrm{e}_{\mathrm{i}}$ represents the end berthing time of vessel $i$; $s_{i}^{+}(w), s_{i}^{-}(w)$ represent the increment and decrement of $s_{i}$ in scenario $w$ respectively; $y_{i}^{+}(w), y_{i}^{-}(w)$ represent the increment and decrement of $y_{i}$ in scenario $w$ respectively; $e_{i}^{+}(w), e_{i}^{-}(w)$ represent the increment and decrement of $e_{i}$ in scenario $w$ respectively; $s_{i}^{\Delta+}(w), s_{i}^{\Delta-}(w)$ represent the increment and decrement with represent to $\left(s_{i}-\mathrm{EAT}_{\mathrm{i}}\right)$ in scenario $w$ respectively; $y_{i}^{\Delta+}(w), y_{i}^{\Delta-}(w)$ represent the increment (or decrement) with respect to $\left|y_{i}-\mathrm{b}_{\mathrm{i}}\right|$ in scenario $w$ respectively; $e_{i}^{\Delta+}(w), e_{i}^{\Delta-}(w)$ represent the increment (or decrement) with respect to $\left(e_{i}-E F T_{i}\right)$ in scenario $w$ respectively; $\triangle b_{i}$ represents the segment deviation of vessel $i$ between the actual berthing position and preferred berthing position; $\triangle b_{i}(w)$ represents the segment deviation of vessel $i$ between the actual berthing position and preferred berthing position in scenario $\omega ; X_{i j}$ is $0-1$ decision variable, $X_{i j}=1$, if Vessel $i$ is located in the left of Vessel $j$ in the 2-dimensional berth-time plane; $X_{i j}=0$, otherwise; $Y_{i j}$ is $0-1$ decision variable, $Y_{i j}=1$, if Vessel $i$ is located below Vessel $j$ in the 2-dimensional berth-time plane; $Y_{i j}=0$, otherwise; $X_{i j}(w)$ is 0 1 decision variable, $X_{i j}=1$, if Vessel $i$ is located in the left of Vessel $j$ in the 2dimensional berth-time plane in scenario $w ; X_{i j}=0$, otherwise; $Y_{i j}(w)$ is $0-1$ decision variable, $Y_{i j}=1$, if Vessel $i$ is located below Vessel $j$ in the 2-dimensional berth-time plane in scenario $\mathrm{w} ; Y_{i j}=0$, otherwise; $r_{i t}$ is $0-1$ decision variable, $r_{i t}=1$, if at least one QC is assigned to vessel $i$ in period $\mathrm{t} ; r_{i t}=0$, otherwise; $r_{i t}(w)$ is $0-1$ decision variable, $r_{i t}=1$, if at least one QC is assigned to vessel $i$ in period $\mathrm{t}$ in scenario $\mathrm{w}$; $r_{i t}=0$, otherwise; $r_{i t q}$ is $0-1$ decision variable, $r_{i t q}=1$, if $q$ QCs are assigned to 
vessel $i$ in period t; $r_{i t q}=0$, otherwise; $r_{i t q}(w)$ is $0-1$ decision variable, $r_{i t q}=1$, if $q$ QCs are assigned to vessel $i$ in period $\mathrm{t}$ in scenario $w ; r_{i t q}=0$, otherwise.

\subsection{Mathematical model}

The objective function of the model is to minimize the sum of waiting time and delayed finishing time of baseline schedule and the expected value of the adjusting time in each scenarios.

$$
\begin{aligned}
& \min f=\sum_{i=1}^{N}\left[\left(s_{i}-E T A_{i}\right)+\left(e_{i}-E T D_{i}\right)\right] \\
& +\sum_{w=1}^{W}\left\{p(w) \sum_{i=1}^{N}\left[\Delta s_{i}^{+}(w)-\Delta s_{i}^{-}(w)+\Delta e_{i}^{+}(w)-\Delta e_{i}^{-}(w)\right]\right\} \\
& e_{i} \leq s_{j}+M\left(1-X_{i j}\right), \quad \forall i, j \in V, i \neq j \\
& \mathrm{y}_{i}+l_{i} \leq y_{j}+M\left(1-Y_{i j}\right), \quad \forall i, j \in V, i \neq j \\
& X_{i j}+Y_{i j}+X_{j i}+Y_{j i} \geq 1, \quad \forall i, j \in V, i \neq j \\
& y_{i}+l_{i} \leq L, \quad \forall i \in V \\
& s_{i} \geq E T A_{i}, \quad \forall i \in V \\
& \sum_{i \in V} \sum_{q \notin Q} q \cdot \varphi_{i t q} \leq|Q|, \quad \forall t \in T \\
& \sum_{q \in Q} q \cdot \varphi_{i t q} \leq R_{i}^{\max } \cdot \zeta_{i t}, \quad \forall i \in V, t \in T \\
& \sum_{q \in Q} q \cdot \varphi_{i t q} \geq R_{i}^{\mathrm{min}} \cdot \zeta_{i t}, \quad \forall i \in V, t \in T \\
& \sum_{q \in Q} \varphi_{i t q}=\zeta_{i t}, \quad \forall i \in V, t \in T \\
& \zeta_{i t} \cdot(t+1) \leq e_{i}, \quad \forall i \in V, t \in T \\
& \zeta_{i t} \cdot t+M \cdot\left(1-\zeta_{i t}\right) \geq s_{i}, \quad \forall i \in V, t \in T \\
& \sum_{k=1}^{B} \Delta b_{i k} \cdot k \geq\left|y_{i}-b_{i}\right|, \quad \forall i \in V \\
& \sum_{k=1}^{B} \Delta b_{i k} \leq 1, \quad \forall i \in V \\
& \sum_{t \in T} \sum_{q \in Q} q \cdot \varphi_{i t q} \cdot \gamma_{q} \geq \frac{m_{i}}{\mu} \cdot \Delta b_{i}^{0.14}, \quad \forall i \in V \\
& \sum_{t \in T} \sum_{q \notin Q} q \cdot \varphi_{i t q} \cdot \gamma_{q} \geq \frac{m_{i}}{\mu}, \quad \forall i \in V \\
& \sum_{t \in T} \zeta_{i t}=e_{i}-s_{i}, \quad \forall i \in V
\end{aligned}
$$




$$
\begin{aligned}
& e_{i}+e_{i}^{+}(w)-e_{i}^{-}(w) \leq s_{j}+s_{j}^{+}(w)-s_{j}^{-}(w)+M\left[1-X_{i j}(w)\right], \quad \forall i, j \in V, i \neq j, w \in \Omega \\
& y_{i}+y_{i}^{+}(w)-y_{i}^{-}(w)+l_{i} \leq y_{j}+y_{j}^{+}(w)-y_{j}^{-}(w)+M\left[1-Y_{i j}(w)\right], \quad \forall i, j \in V, i \neq j, w \in \Omega \\
& X_{i j}(w)+Y_{i j}(w)+X_{j i}(w)+Y_{j i}(w) \geq 1, \quad \forall i, j \in V, i \neq j, w \in \Omega \\
& l_{i} \leq y_{i}+y_{i}^{+}(w)-y_{i}^{-}(w)+l_{i} \leq L, \quad \forall i \in V, w \in \Omega \\
& s_{i}+s_{i}^{+}(w)-s_{i}^{-}(w) \geq E T A_{i}(w), \quad \forall i \in V, w \in \Omega \\
& s_{i}-E T A_{i}+\Delta s_{i}^{+}(w)-\Delta s_{i}^{-}(w)=s_{i}+s_{i}^{+}(w)-s_{i}^{-}(w)-E T A_{i}(w), \quad \forall i \in V, w \in \Omega \\
& \left|y_{i}-b_{i}\right|+\Delta y_{i}^{+}(w)-\Delta y_{i}^{-}(w)=\left|y_{i}+y_{i}^{+}(w)-y_{i}^{-}(w)-b_{i}\right|, \quad \forall i \in V, w \in \Omega \\
& e_{i}-\operatorname{ETD}_{i}+\Delta e_{i}^{+}(w)-\Delta e_{i}^{-}(w)=e_{i}+e_{i}^{+}(w)-e_{i}^{-}(w)-\operatorname{ETD}_{i}(w), \quad \forall i \in V, w \in \Omega \\
& \sum_{i \in V} \sum_{q \notin Q} q \cdot \varphi_{i t q}(w) \leq|Q|, \quad \forall t \in T, w \in \Omega \\
& \sum_{q \in Q} q \cdot \varphi_{i t q}(w) \leq R_{i}^{\max }(w) \cdot \zeta_{i t}(w), \quad \forall i \in V, t \in T, w \in \Omega \\
& \sum_{q \in Q} q \cdot \varphi_{i t q}(w) \geq R_{i}^{\min }(w) \cdot \zeta_{i t}(w), \quad \forall i \in V, t \in T, w \in \Omega \\
& \sum_{q \in Q} \varphi_{i t q}(w)=\zeta_{i t}(w), \forall i \in V, t \in T, w \in \Omega \\
& \zeta_{i t}(w) \cdot(t+1) \leq e_{i}(w)+e_{i}^{+}(w)-e_{i}^{-}(w), \quad \forall i \in V, t \in T, w \in \Omega \\
& \zeta_{i t}(w) \cdot t+M \cdot\left[1-\zeta_{i t}(w)\right] \geq s_{i}+s_{i}^{+}(w)-s_{i}^{-}(w), \quad \forall i \in V, t \in T, w \in \Omega \\
& \sum_{k=1}^{B} \Delta b_{i k}(w) \cdot k \geq\left|y_{i}(w)+y_{i}^{+}(w)-y_{i}^{-}(w)-b_{i}\right|, \quad i \in V, w \in \Omega \\
& \sum_{k=1}^{B} \Delta b_{i k}(w) \leq 1, \quad i \in V, w \in \Omega \\
& \sum_{t \in T} \sum_{q \in Q} q \cdot \varphi_{i t q}(\omega) \cdot \gamma_{q} \geq \Delta b_{i}(\omega)^{0.14} \frac{m_{i}(\omega)}{\mu}, \quad i \in V, \omega \in \Omega \\
& \sum_{t \in T} \sum_{q \notin Q} q \cdot \varphi_{i t q}(w) \cdot \gamma_{q} \geq \frac{m_{i}(w)}{\mu}, \quad i \in V, w \in \Omega \\
& \sum_{t \in T} \zeta_{i t}(w)=e_{i}+e_{i}^{+}(w)-e_{i}^{-}(w)-\left[s_{i}+s_{i}^{+}(w)-s_{i}^{-}(w)\right], \quad \forall i \in V, w \in \Omega \\
& \Delta b_{i}=\left|y_{i}-b_{i}\right| / L_{s}, \quad \forall i \in V \\
& X_{i j}, Y_{i j}, X_{i j}(w), Y_{i j}(w) \in\{0,1\}, \quad \forall i, j \in V, w \in \Omega
\end{aligned}
$$




$$
\begin{aligned}
& \zeta_{i t}, \varphi_{i t q}, \zeta_{i t}(w), \varphi_{i t q}(w) \in\{0,1\}, \quad \forall i \in V, t \in T, q \in Q, w \in \Omega \\
& \Delta b_{i}, \Delta b_{i}(w) \in\{0,1\}, \quad \forall i \in V, w \in \Omega \\
& s_{i}(w), s_{i}^{+}(w), s_{i}^{-}(w), e_{i}(w), e_{i}^{+}(w), e_{i}^{-}(w) \geq 0, \quad \forall i \in V, w \in \Omega \\
& \Delta s_{i}^{+}(w), \Delta s_{i}^{-}(w), \Delta e_{i}^{+}(w), \Delta e_{i}^{-}(w), y_{i} \geq 0, \forall i \in V, w \in \Omega
\end{aligned}
$$

Constraints (2)-(3) define the berth position and order of any two vessels. Constraint (4) ensures there is no overlap among all vessels in the 2-dimensional berth-time plane. Constraint (5) ensures the positions of all vessels are restricted by the length of the terminal. Constraint (6) implies that the start berthing time cannot earlier than the expected arrival time. Constraint (7) ensures that the number of QCs assigned to all vessels cannot exceed the total number of available QCs in any time segments. Constraints (8)-(9) ensure that the number of QCs assigned to a vessel must not be greater than the maximum number of QCs allowed to serve simultaneously, and not be smaller than the minimum number of QCs should be assigned. Constraint (10) determines the relationship between $r_{i t q}$ and $r_{i t}$. Constraint (11) ensures that there is no QC assigned to vessel $i$ after it departs. Constraint (12) ensures that there is no QC is assigned to vessel $i$ before it arrives. Constraints (13) defines the number of berth deviation segments of vessel $i$. Constraint (14) ensures that the number of berth deviation segments of vessel $i$ takes only one specific value. Constraints (15) ensure that QC assignments for a vessel must satisfy the vessel's real QC hours demand considering the QCs' idle times as the result of berthing deviation. Constraint (16) ensures that QC assignments for a vessel must satisfy the vessel's QC hours demand without the berthing deviation. Constraint (17) determines the relationship between the handling time and the start berthing time or the end berthing time. Constraints (18)-(19) define the berth position and order of any two vessels after adjusting vessels' schedules. Constraint (20) implies there is no overlap among all vessels in the 2-dimensional berth-time plane after adjusting vessels' schedules. Constraint (21) ensures the positions of all vessels are restricted by the length of the terminal in varied scenario. Constraint (22) implies that the newly planned start berthing time cannot earlier than the expected arrival time. Constraint (23) builds the relationship between the adjustments of start berthing time $\left(s_{i}^{+}(w), s_{i}^{-}(w)\right)$ and the change of waiting time $\left(s_{i}^{\Delta+}(w), s_{i}^{\Delta-}(w)\right)$. Constraint (24) builds the relationship between the adjustments of actual berthing position $\left.\left(y_{i}^{+}(w), y_{i}^{-}(w)\right)\right)$ and the change of deviation from the best berthing position $\left(y_{i}^{\Delta+}(w), y_{i}^{\Delta-}(w)\right)$. Constraint (25) builds the relationship between the adjustments of end berthing time $\left(e_{i}^{+}(w), e_{i}^{-}(w)\right)$ and the change of delayed finishing time $\left(e_{i}^{\Delta+}(w), e_{i}^{\Delta-}(w)\right)$. Constraint (26)-(27) ensure that the newly planned number of QCs assigned to a vessel must not be greater than the maximum number and not be smaller than the minimum number of QCs should be assigned. Constraint (28) ensures that the newly planned number of QCs assigned to all vessels cannot exceed the total number of available QCs in any time segments. Constraint (29) determines the relationship between $r_{i t q}(w)$ and $r_{i t}(w)$. Constraint (30) ensures that there is no QC assigned to vessel $i$ after it departs. Constraint (31) ensures that there is no QC assigned to vessel $i$ before it arrives. Constraint (32) defines the newly planned number of berth deviation segments of vessel $i$. Constraint (33) ensures that the newly planned number of berth deviation segments of vessel $i$ takes only one specific value. Constraint (34) ensure that QC assignments for a vessel must satisfy the vessel's real QC hours demand considering 
berthing deviation in varied scenario. Constraint (35) ensures that QC assignments for a vessel must satisfy the vessel's QC hours demand without the berthing deviation in varied scenario. Constraint (36) determines the relationship between handling time and newly planned actual start berthing time or actual end berthing time. Constraints (37) calculates the deviation of berthing position. (38)-(42) define the integer or binary variables.

\section{Numerical experiments}

To verify the effectiveness of the proposed model, we compare the results between the proposed model (M1) and the traditional model (M2), which only the proactive strategy is used (the recovery schedule is not included). Table 1 and Table 2 are the comparison results with vessels delay and containers increased respectively, and it shown that the proposed model has a better efficiency to deal with uncertainties.

Table 1. Results with vessels delay.

\begin{tabular}{ccccccc}
\hline$|V|$ & $|\Omega|$ & Delayed Vessels & Delay time & M1 & M2 & $G A P(\%)$ \\
\hline 8 & 5 & 1 & $1 \mathrm{~h}$ & 390 & 450 & 15.38 \\
8 & 5 & 2 & $1 \mathrm{~h}$ & 420 & 480 & 14.29 \\
8 & 5 & 3 & $1 \mathrm{~h}$ & 450 & 510 & 13.33 \\
8 & 5 & 1 & $2 \mathrm{~h}$ & 420 & 480 & 14.29 \\
8 & 5 & 2 & $2 \mathrm{~h}$ & 480 & 540 & 12.50 \\
8 & 5 & 3 & $2 \mathrm{~h}$ & 540 & 600 & 11.11 \\
8 & 5 & 1 & $3 \mathrm{~h}$ & 450 & 510 & 13.33 \\
8 & 5 & 2 & $3 \mathrm{~h}$ & 540 & 600 & 11.11 \\
8 & 5 & 3 & $3 \mathrm{~h}$ & 630 & 690 & 9.52 \\
\hline
\end{tabular}

Table 2. Results with vessel containers increased.

\begin{tabular}{ccccccc}
\hline$|V|$ & $|\Omega|$ & Altered Vessels & Altered volume & M1 & M2 & GAP $(\%)$ \\
\hline 8 & 5 & 0.10 & 40 & 360 & 420 & 16.67 \\
8 & 5 & 0.10 & 40 & 360 & 420 & 16.67 \\
8 & 5 & 0.10 & 40 & 360 & 420 & 16.67 \\
8 & 5 & 0.10 & 80 & 372 & 432 & 16.13 \\
8 & 5 & 0.10 & 80 & 378 & 438 & 15.87 \\
8 & 5 & 0.10 & 80 & 402 & 462 & 14.93 \\
8 & 5 & 0.10 & 120 & 390 & 450 & 15.38 \\
8 & 5 & 0.10 & 120 & 426 & 486 & 14.08 \\
8 & 5 & 0.10 & 120 & 450 & 510 & 13.33 \\
\hline
\end{tabular}




\section{Conclusions}

This paper studied the integrated optimization of BAP and QCAP under uncertainties. A stochastic programming model is formulated for minimizing the waiting time and delay departure time of vessels. Besides, numerical experiments and scenario analysis are conducted to validate the effectiveness of the proposed model. The results can help port operators to generate a better schedule for dealing with the impact of various uncertainties. Furthermore, it will improve operational efficiency, reduce operating costs and bring economic benefit to terminal operators. However, there are still some limitations in this paper. For example, the increase of uncertain parameters will increase the scale of the experiment in geometric series. The scale of the experiment is also limited to small-scale experiments. In addition to the above factors, we will concern the impact of the probability fluctuation of the scene on the plan as well in the future.

\section{Acknowledgement}

This work is sponsored by the National Natural Science Foundation of China [grant number 71602114, 71974122]; Shanghai Science \& Technology Committee Research Project [grant number 17040501700]; Shanghai Rising-Star Program [grant number 19QA1404200], Shanghai Sailing Program [grant number 19YF1418800] and Shanghai Special Research Project [grant number 17DZ2280200].

\section{References}

[1] Drewry Shipping Consultants, Container Forecaster Quarter 1·2020, London, 2020.

[2] J.-L. He, Berth allocation and quay crane assignment in a container terminal for the trade-off between timesaving and energy-saving, Advanced Engineering Informatics, 2016, Vol. 30, pp. 390-405.

[3] C. Bierwirth and F. Meisel, A survey of berth allocation and quay crane scheduling problems in container terminals, European Journal of Operational Research, 2009, Vol. 202, pp. 615-627.

[4] C. Bierwirth and F. Meisel, A follow-up survey of berth allocation and quay crane scheduling problems in container terminals, European Journal of Operational Research, 2015, Vol.244, pp. 675-689.

[5] Z.-X. Wang and C.-H. Guo, Minimizing the risk of seaport operations efficiency reduction affected by vessel arrival delay, Industrial Management \& Data Systems, 2018, Vol. 18, pp. 1498-1509.

[6] L. Zhen, L.-H. Lee and E.-P. Chew. A decision model for berth allocation under uncertainty, European Journal of Operational Research, 2011, Vol. 212, pp. 54-68.

[7] X.-L. Han, Z.-Q. Lu and L.-F Xi. A proactive approach for simultaneous berth and quay crane scheduling problem with stochastic arrival and handling time, European Journal of Operational Research, 2010, Vol. 207, pp. 1327-1340.

[8] M. Hendriks, M. Laumanns, E. Lefeber, et al, Robust cyclic berth planning of container vessels, $O R$ Spectrum, 2010, Vol. 32, pp. 501-517.

[9] M. Golias, I. Portal, D. Konur, et al, Robust berth scheduling at marine container terminals via hierarchical optimization, Computers \& Operations Research, 2014, Vol. 41, pp. 412-422.

[10] R.-M. Mario, P.-I. Laura, B. Federico, et al, A genetic algorithm for robust berth allocation and quay crane assignment, Progress in Artificial Intelligence, 2014, Vol. 2, pp. 177-192.

[11] T.-S. Xiao, J.-X. Cao and R. Jie, A robust optimization approach to the integrated berth allocation and quay crane assignment problem, Transportation Research Part E: Logistics and Transportation Review, 2016, Vol. 94, pp. 44-65.

[12] Q.-C. Zeng, Z.-Z. Yang and X.-P. Hu, Disruption recovery model for berth and quay crane scheduling in container terminals, Engineering Optimization, 2011, Vol. 43, pp. 967-983.

[13] M.-Z. Li, J.-G. Jin and C.-X. Lu, Real-Time Disruption Recovery for Integrated Berth Allocation and Crane Assignment in Container Terminals, Transportation Research Record, 2015, issue 2479, pp. 49-59.

[14] X. Xiang, C.-C Liu, and L.-X. Miao, Reactive strategy for discrete berth allocation and quay crane assignment problems under uncertainty, Computers \& Industrial Engineering, 2018, Vol. 126, pp. 196-216. 